Arteterapia. Papeles de arteterapia y educación para inclusión social ISSN-e 1988-8309

http://dx.doi.org/10.5209/ARTE.59779

\title{
Vulnerabilidad, ruptura social y arteterapia
}

\author{
Eva Mayo $^{1}$
}

Recibido: 3 de abril de 2018/ Aceptado: 17 de julio de 2018

Resumen. Desde la vulnerabilidad social hasta la exclusión social la persona hace frente a un proceso de continuas rupturas y problemáticas difíciles, cuando no imposibles, de superar en soledad. Ante la pérdida del vinculo social y familiar, soporte emocional y factor de protección fundamental para sobrellevar la desventaja social, una intervención social capacitadora y reparadora es esencial. Con este artículo, se realiza una aproximación a las oportunidades que ofrece el arteterapia en el ámbito social para una intervención preventiva, en el marco de la tendencia de trabajo interdisciplinar que se viene siguiendo en el contexto de los servicios sociales. De la confluencia de los objetivos propios de la intervención social y el arteterapia, encontramos estrategias que mejoran la vida de las personas. Les proporcionan bienestar y les facilitan movilizar los recursos propios para el afrontamiento de las rupturas, que en sus distintas dimensiones, personal, social, económico y cultural, resultan ser experiencias traumáticas que se suceden en sus trayectorias vitales.

Palabras clave: Arteterapia; Exclusión Social; Intervención Social; Prevención; Vulnerabilidad

\section{[en] Vulnerability, social rupture and art therapy}

\begin{abstract}
From social vulnerability to social exclusion, the person faces a process of continuous ruptures and difficult, if not impossible, problems to overcome in solitude. Faced with the loss of social and family ties, emotional support and a fundamental protection factor to overcome social disadvantage, a social intervention that is both training and repairing is essential. With this article, an approach is made to the opportunities offered by art therapy in the social field for a preventive intervention, within the framework of the interdisciplinary work trend that is being followed in the context of social services. From the confluence of the objectives of social intervention and art therapy, we find strategies that improve people's lives. They provide welfare and help them mobilize their own resources to cope with the ruptures, which in their different dimensions, personal, social, economic and cultural, turn out to be traumatic experiences that occur in their life trajectories.
\end{abstract}

Keywords: Art therapy; Social exclusion; Social intervention; Prevention; Vulnerability

Sumario. 1. Introducción. La exclusión social como proceso multidimensional. 2. Vulnerabilidad, ruptura y afrontamiento. 3. Atención social interdisciplinar y arteterapia. 4. Aportaciones del Arteterapia al desarrollo personal y social. 5. Aportaciones del arteterapia al desarrollo la intervención social. 6 . Conclusiones 7. Referencias bibliográficas.

Cómo citar: Mayo, E. (2018). Vulnerabilidad, ruptura social y arteterapia, en Arteterapia. Papeles de arteterapia y educación para inclusión social 13, 2018, 33-52.

1 masgente@hotmail.com 


\section{Introducción. La exclusión social como proceso multidimensional}

Nos encontramos en un contexto marcado por la complejidad en los ejes de la desigualdad, donde se consolida una sociedad dual compuesta por personas incluidas y excluidas en el sistema y un acceso al bienestar cada vez más mercantilizado.

La crisis económica ha acentuado la desigualdad incrementando los colectivos vulnerables a la exclusión social. Ahora, la marginación ya no solo encuentra su origen en situaciones específicas, como el paro, las malas condiciones de salud o la baja formación que impidan alcanzar unas condiciones de vida mínimas. A los colectivos de riesgo tradicionales (personas inmigrantes, mujeres, discapacitadas, etc.), como señala Castel (2014), se incorporan personas que, hasta el momento, habían podido integrarse en la sociedad y pasan de haber sido capaces de gestionar su vida, a sentir la incertidumbre de un futuro que no pueden controlar. El riesgo de marginación se ha ampliado, y la pobreza ya no puede limitarse a la noción tradicional asociada a la falta de recursos materiales.

La desventaja social se ha convertido en un problema que tiene dimensiones económicas, sociales, psicológicas y culturales que no es posible separar. Una desventaja que lleva a las personas a una situación de vulnerabilidad que es dinámica, multifactorial y multidimensional (Subirats et al., 2005; Hernández, 2008). La exclusión social ya no es entendida como una situación. Se trata de un proceso, un camino en el que la persona encadena rupturas sucesivas (Castel, 2005) perdiendo el sentimiento de pertenencia a la sociedad y aumentando con ello su vulnerabilidad (Sánchez y Jiménez, 2013). Para Subirats et al. (2005) «la exclusión social es el resultado de una cadena de acontecimientos y condiciones estructurales que han desconectado a multitud de personas de las diferentes esferas de la sociedad».

Cuando Castel (2014) nos habla de la gran transformación de los procesos de exclusión social, asegura que los problemas que plantea la exclusión ya no se encuentran únicamente en situarse en fuera o dentro de los márgenes de la sociedad, sino que responde a múltiples variables y factores y la combinación entre sí, que da como resultado circunstancias individuales muy distintas.

En el marco de una sociedad «liquida» (Bauman, 2005), donde dinámicas socioeconómicas y culturales son cambiantes y globales, encontramos que las estructuras colectivas son frágiles y las relaciones sociales débiles. El papel de la familia como proveedor de bienestar y red de apoyo social se va diluyendo en favor de una sociedad más individualista. Esa circunstancia, unida a las trayectorias vitales que ya no son lineales, sino inestables y erráticas, lleva a las personas a caminar afrontando las dificultades y la incertidumbre desde la individualidad.

Necesariamente este enfoque afecta a las estrategias de trabajo en el ámbito social que, con el deseo de proporcionar bienestar con sus actuaciones no puede tener como objetivo único la restauración del acceso a los bienes materiales para la supervivencia. Por sí solos, los recursos económicos ya no son garantía de protección ni de integración a medio y largo plazo, hay más factores que considerar que garanticen la participación de las personas en la comunidad. Al analizar las circunstancias vitales y enfocar la intervención, se ha de tener en consideración la dimensión relacional y el impacto de los eventos socioeconómicos en las situaciones de vulnerabilidad. 


\section{Vulnerabilidad, ruptura y afrontamiento}

La vulnerabilidad es una condición social de riesgo, una dificultad que impide a las personas alcanzar el bienestar, en relación a la tanto a la subsistencia como a la calidad de vida (Malgesini et al., 2007). Al hablar de vulnerabilidad social, nos referimos a una franja que se encuentra entre la zona de integración y la zona de exclusión, entre los recursos económicos, sociales y familiares estables y el aislamiento social y la carencia. (IGOP, 2003; Malgesini, 2011). Es una zona gris que matiza la dualidad exclusión-inclusión, un territorio por el que discurre el proceso que lleva a las personas, familias y comunidades a estar en riesgo de encontrarse en los límites del sistema social. Afirma Castel (2002) que la vulnerabilidad social es una zona intermedia, inestable, que conjuga la precariedad del trabajo y la fragilidad de los soportes de proximidad.

Vemos como, por un lado, la vulnerabilidad social se encuentra vinculada, como señala Pizarro (2001), a la «inseguridad e indefensión que experimentan las comunidades, grupos, familias e individuos en sus condiciones de vida» como consecuencia «del impacto provocado por algún tipo de evento natural, económico y social de carácter traumático». Resulta un enfoque interesante, especialmente si lo ponemos en relación con la idea que plantea Castel (2002) sobre la exclusión social. Para Castel, trata de un proceso dinámico que encadena rupturas sucesivas que llevan al aislamiento, un proceso de «desafiliación $»^{2}$ mediante el cual la persona se va desligando de las redes de proximidad que permiten su protección e integración social. Estas rupturas producen impactos, que pueden resultar traumáticos del modo que apunta Pizarro, generando inseguridad e indefensión.

Por otro lado, la vulnerabilidad social también se encuentra asociada a la capacidad de las comunidades, familias y personas para gestionar los recursos y manejar las estrategias que permitan hacer frente a los eventos traumáticos. Las dificultades de autoajuste ante los cambios del medio ambiente de una persona o comunidad constituyen un riesgo (Ander-Egg, 2009) cuyo origen encontramos en factores físicos, socioeconómicos y sociopolíticos. El desempleo, la enfermedad, la falta de hogar, analfabetismo, discapacidad, ser mujer, mayor, la pertenencia a un colectivo inmigrante, el entorno, la falta de red social, etc.... son aspectos que impactan en las personas, que son un riesgo. La fragilidad se enfatiza en la incapacidad que manifiestan las personas, familias o los grupos para enfrentarse y neutralizar las situaciones traumáticas que experimentan. La vulnerabilidad se encuentra relacionada no solo una problemática en sí misma, sino también con la vivencia individual de la situación y su capacidad de afrontarla. En el presente, o por los efectos derivados que puedan sobrevenir en el futuro, los riesgos pueden ocasionar una sucesión rupturas y pérdidas que alejan a las personas de la zona de integración.

La vulnerabilidad es un fenómeno con múltiples dimensiones, donde la exposición a los factores de riesgo y la habilidad para encarar las situaciones problemáticas determinan el grado de vulnerabilidad de una persona, familia o grupo social. Esto es, la incapacidad para enfrentar las amenazas, las dificultades para adaptarse activa-

«desafiliación»: proceso mediante el cual la persona se encuentra disociada de las redes sociales y societales que permiten su protección de las eventualidades de la vida. Habla de desafiliación con el fin de explicar mejor los procesos que llevan a las personas a las distintas zonas de vulnerabilidad. La utilización del concepto desafiliación hace referencia a un recorrido, frente al concepto exclusión que sugiere inmovilidad como si no hubiera matices en la vulnerabilidad. (Castel, 2002) 
mente a las situaciones, y en definitiva, carecer de recursos para afrontar sus efectos representa una fragilidad recogida en diversos estudios sobre la vulnerabilidad social (Hernández, 2008; Malgesini, 2011; Pizarro, 2001; Flores, 2014; Kaztman, 2000).

Diversidad de trabajos ponen en valor la red social a la hora analizar la vulnerabilidad. La carencia de ésta contribuye a profundizar en la desprotección y consolidar el proceso de aislamiento social. Retomando el enfoque de Castel, en el que se considera la nueva pobreza en torno a los ejes del empleo y la sociabilidad, encontramos en la escasez relacional un indicador de vulnerabilidad que interviene en el proceso de desvinculación de la sociedad. Pizarro (2001), por su parte, también destaca la importancia de las relaciones sociales. Se refiere a estas como «Los vínculos y redes que tienen las personas y familias son muy determinantes para acceder a oportunidades de trabajo, información y posiciones de poder.» Ruiz (2011), así mismo, destaca el componente social del fenómeno.

Las redes sociales representan unos recursos básicos para afrontar las vivencias traumáticas, un apoyo para contrarrestar los efectos negativos de los impactos vitales, para superar la inseguridad, los riesgos y el estrés de encontrarse en la zona de la vulnerabilidad. En un contexto donde los lazos solidarios que proporciona la familia y el contexto inmediato son escasos o inexistentes, cuando los recursos del sistema resultan inaccesibles para las personas, es necesario buscar mecanismos de apoyo en el presente, y de protección para el futuro. Hay que tener en cuenta que el riesgo de exclusión correlaciona con la falta de red social y familiar. El aislamiento social y/o familiar tiene consecuencias tanto psicológicas como materiales que pueden colocar a quienes los padecen en una situación de vulnerabilidad extrema, desplazándolos rápidamente hacia el terreno de la exclusión.

La atención que pone Flores $^{3}$ (2014) en los efectos subjetivos y emocionales de la vulnerabilidad, nos lleva a considerar también la importancia de la atención a la individualidad y la relevancia de incorporar en la intervención social la dimensión emocional. No en vano, la perdida del sentimiento de pertenencia a la comunidad y junto a la fragilidad relacional puede intensificar la vulnerabilidad social sea cual sea el origen principal de ésta como señalaban Subirats et al. (2005). Que las personas encuentren la forma de gestionar la tensión, la incertidumbre y la indefensión que padecen ante las perdidas y las rupturas que originan las circunstancias socioeconómicas es un recurso para el bienestar que el trabajo social asume. Que el afrontamiento de estas vivencias traumáticas no solo debe considerar la dimensión individual, sino que requiere de un fortalecimiento de las redes sociales de proximidad, un mantener o generar cierto «capital relacional» (Alvarez-Uria, 2009) que limite el choque emocional que implica la vulnerabilidad social.

\section{Atención social interdisciplinar y arteterapia}

Los enfoques tradicionales del Trabajo Social se encuentran más centrados en el diagnostico de los problemas, en determinar las deficiencias y la incapacidad de las personas o familias para adaptarse a las circunstancias y sobrellevar las condiciones de vida adversas. Cuando se hace referencia, por ejemplo, a familias inmersas en

3 En su estudio Flores pone el foco en el análisis del efecto subjetivo y emocional que presentan mujeres migrantes en una comunidad en situación de pobreza extrema en el Pañuelo, México. 
procesos de exclusión, no se alude a una única dificultad sino que responden habitualmente a problemáticas múltiples (violencia, desempleo, desestructuración, falta de habilidades parentales, etc.). Siendo cierto que sus integrantes suelen encontrarse en situaciones particularmente adversas, la tendencia en la intervención social es estructurar la intervención poniendo el foco en las fortalezas de las personas para afrontar los retos vitales. (Segado, 2011; De la paz, Rodríguez y Mercado, 2014). Las personas y las familias objeto de la atención social son, a menudo, grupos que se ven en la necesidad de sobrevivir inmersos en experiencias traumáticas, en riesgos o procesos de exclusión, con dificultades para acceder a los recursos básicos. Como señala Segado (2011), superar estas circunstancias requiere familias extraordinariamente resilientes.

Como apuntan algunos estudios (Rodríguez, Gómez y Ramírez, 2017; Hernández-Echegaray, 2016) en las tendencias del trabajo social, la atención social se plantea como reto realizar intervenciones más centradas en la persona y su entorno. De este modo, la persona que acude a los servicios sociales ya nos es dirigida por un itinerario de inserción propuesto por un/a profesional, sino que ella es un elemento central, toma un papel protagonista en su proceso de cambio, creando su propia historia y poniendo en juego sus fortalezas personales para adquirir control sobre su propio presente y su futuro. Y activando factores de protección como la resiliencia para así obtener una respuesta positiva a los efectos adversos de la vulnerabilidad social.

En su revisión de los planteamientos del trabajo social como práctica, los estudios anteriormente señalados entre otros, se plantean superar la excesiva burocratización y el enfoque asistencialista en el ámbito de los servicios sociales, ya que esto limita la intervención social a meros trámites administrativos y que los servicios sociales sean considerados únicamente un sistema de provisión de recursos materiales. Así mismo, si bien el trabajo social ha recurrido de forma natural a otras disciplinas para realizar consultas sobre asuntos específicos durante una intervención, se ha visto la necesidad de impulsar el objetivo ideal del trabajo interdisciplinar y en red. Una metodología que pronostica el enriquecimiento mutuo de las disciplinas, y una mejor respuesta a los problemas sociales multidimensionales manteniendo una visión integral del problema.

Como hemos visto, en el ámbito de los servicios sociales la tendencia en la práctica profesional es orientar sus actuaciones con un enfoque integral, centrado en la persona e interdisciplinar. Con esta vocación, los recursos tradicionales son ampliados al recurrir a otras disciplinas y a las propias personas usuarias para lograr los objetivos de bienestar y protección social. Al amparo de estos planteamientos, consideramos que el arteterapia proporciona bases suficientes como para ser tenida en cuenta como una herramienta válida que contribuye a reforzar los objetivos socioeducativos de prevención e intervención en el ámbito del trabajo social. Así mismo, el espacio del arteterapia se convierte en un espacio de observación que permite obtener información valiosa para la detección de situaciones de vulnerabilidad como pudimos verificar en un estudio de relacionado con un Servicio de Infancia, Juventud y Familia de los Servicios Sociales de Base de un municipio de Bizkaia donde se realizaban talleres de arteterapia.

La intervención arteterapéutica permite a las personas elaborar sus vivencias y expresar sus inquietudes a través del lenguaje plástico, especialmente adecuado cuando con los impactos traumáticos bloquean la comunicación verbal. El artetera- 
pia realiza una indagación personal «intensa y transformadora» (Lund, 2014) ya que, con la creatividad, la persona es capaz de desarrollar estrategias más flexibles y más ajustadas a la realidad, y adaptarse aunque esta cambie, lo que permite hacer frente a la vulnerabilidad. Consolidando las estrategias adquiridas a través del arteterapia, se refuerza una intervención menos asistencialista y más capacitadora, acorde a las tendencias actuales del trabajo social.

Considerando que el arteterapia constituye un recurso válido y reparador para las personas en situación de fragilidad, nos proponemos indagar las posibilidades de incorporar esta disciplina en el ámbito de los Servicios Sociales de Base. Para ello analizamos su encaje poniendo en relación los objetivos de desarrollo social y personal compartidos por las disciplinas, arteterapia y trabajo social. Por ello recogemos las aportaciones de distintos estudios y trabajos sobre arteterapia, que nos proporcionan una idea de la contribución que puede suponer la disciplinar en el ámbito social, poniendo en relación estos beneficios con el propósito socioeducativo de proporcionar apoyo del proceso de desarrollo físico, afectivo, cognitivo y social de las personas y colectivos en situación de vulnerabilidad. Aunque parte de los logros documentados del arteterapia para el bienestar se refieren a otros ámbitos, consideramos que pueden ser trasferibles al contexto social, en tanto que se abordan objetivos de bienestar personal y la socialización y, desde el punto de vista de la integralidad repercuten positivamente en el desarrollo personal y en la integración social.

\section{Aportaciones del arteterapia a los programas de intervención sociosanitaria}

El arteterapia, en tanto que disciplina en construcción, ofrece un amplio abanico de enfoques de intervención. Sin embargo, tanto la metodología básica como los beneficios que aporta son compartidos por las distintas orientaciones conceptuales (psicoanalítica, humanista, psicopedagógica, ecléctica y multimodal). Los resultados positivos son avalados por diversos estudios en el ámbito educativo, sanitario y social. (Kramer, 1982; Carnacea, 2014; Camarena, 2015; Creux, 2013; Guiote, A. 2013; Moreno, 2012; Kaplan, 2007)

El arteterapia es utilizado en diversidad de centros y servicios de la red social, educativa y de la salud. Encontramos prácticas arteterapéuticas en hospitales, centros de día de salud mental, psiquiátricos, centros de atención a personas con problemas psíquicos, servicios de atención a la infancia en situación de riesgo social, centros de atención a mujeres y menores que han sufrido malos tratos, víctimas de abusos sexuales, en escuelas de educación especial, personas con trastornos alimentarios, con minorías étnicas, con inmigrantes (Moreno, 2006). El colectivo que puede beneficiarse del arteterapia es muy amplio y heterogéneo, resultando una disciplina favorecedora de la atención integral y multidisciplinar que requiere la intervención social.

De acuerdo con González y Gracia (2005), entendemos el trabajo en los ámbitos desfavorecidos requiere de la concurrencia de diversas estrategias de acción que permitan un mayor grado de eficacia a la hora de alcanzar los metas definidas con la persona. Cuando se atienden las necesidades emocionales de las personas que se encuentran en riesgo o exclusión social, se produce una optimización de los programas donde participan. De esto modo, indica Gata (2005), que los programas de modificación de conducta dirigidos a la adquisición de habilidades motoras, cognitivas o sociales pueden verse, en mayor o menor medida, beneficiados si a la vez se atienden debidamente las necesidades emocionales de la persona. 
A continuación se realiza una síntesis de las aportaciones de la intervención arteterapéutica desde distintos ámbitos (social, educativo y sanitario), pero puestos en relación con los objetivos propios de la atención social de contribuir al cambio personal y social que mejore la vida de las personas y permita afrontar las circunstancias adversas y las perdidas traumáticas que acompañan a las personas en situación de vulnerabilidad. Consideramos que está elaboración puede ser de utilidad, en tanto que presenta el arteterapia como disciplina válida para contribuir al contexto especifico de los servicios sociales, y porque converge con las tendencias del trabajo en el ámbito social.

Las contribuciones se han clasificado en torno las áreas de desarrollo personal y social y las aportaciones a la intervención (Gráfico 1). Si bien se presentan de modo diferenciado, sin duda, están inevitablemente relacionadas ya que, por ejemplo, la mejor gestión de las emociones contribuye a las relaciones sociales más habilidosas, lo que ayuda a prevenir las conductas conflictivas y todas ellas facilitan la intervención social.

\begin{tabular}{|c|c|}
\hline \multicolumn{2}{|c|}{ BENEFICIOS DEL ARTETERAPIA } \\
\hline DESARROLLO PERSONAL & $\begin{array}{l}\text { - } \quad \text { Mejora la gestión de las emociones } \\
\text { - Facilita la resolución de problemas } \\
\text { - Ayuda en la superación de experiencias dolorosas } \\
\text { - Disminuye las conductas conflictivas }\end{array}$ \\
\hline DESARROLLO SOCIAL & $\begin{array}{l}\text { - Desarrolla el interés social } \\
\text { - Favorece las relaciones interpersonales } \\
\text { - Mejora las habilidades sociales } \\
\text { - Mejora la expresión y la comunicación }\end{array}$ \\
\hline $\begin{array}{l}\text { DESARRALLO DE LA } \\
\text { INTERVENCIÓN }\end{array}$ & $\begin{array}{l}\text { - Facilita el cambio } \\
\text { - Es una experiencia gratificante } \\
\text { - Facilita la proyección a futuro } \\
\text { - Espacio de detección de necesidades }\end{array}$ \\
\hline
\end{tabular}

Gráfico 1. Beneficios del arteterapia.

Fuente: Elaboración propia

\section{Aportaciones del arteterapia al desarrollo personal y social}

De acuerdo con Callejón (2005), entendemos que los beneficios que el arte como terapia puede proporcionar son claros ya que contribuye al impulso de las capacidades adaptativas, mejora los recursos personales y amplia relaciones sociales. Así mismo, el uso de la terapia artística puede limitar los efectos que las personas sufren fruto las problemáticas sociales. 


\section{a) Área de desarrollo personal}

\section{Mejora la gestión de las emociones}

Vanegas y González (2014) concluyen, corroborando otros estudios, que el lenguaje plástico resulta efectivo para el manejo de las emociones. Duncan (2007) señala que trabajar las emociones con el arteterapia mejora la calidad de las relaciones humanas. Esto se debe a que, centrada en el factor emocional, que es esencial para todas las personas, el arteterapia ayuda a tomar conciencia de las vivencias propias. En relación al trabajo con la infancia, Maite Gata (2005) apunta que el arteterapia puede ser una modalidad de intervención adecuada para que el niño o la niña exprese su angustia y controle sus reacciones emocionales negativas. Esto reduce el impacto que produce una experiencia difícil. Y contribuye a desarrollar estrategias de afrontamiento del estrés.

El proceso artístico es un vehículo donde poder explorarse y desarrollar las habilidades básicas para el óptimo desempeño social (Duncan, 2007). Trabajar con las emociones desde el arteterapia, debe ser un acompañamiento y apoyo al autoconocimiento. Conocer las emociones propias y reconocer las de otras personas (empatía) mejora la facultad para relacionarse (Salovey y Mayer, 1990 citados por Duncan, 2007). La capacidad de expresar la emoción adecuada ante una situación es una habilidad esencial en todas las relacionas cotidianas. La habilidad para manejarse, para adecuarse a las emociones de los demás, determina el grado de capacidad social de la persona.

\section{Facilita la resolución de conflictos}

Edith Kramer defendía que con el proceso creativo el conflicto se reexperimenta desde otra perspectiva, se reelabora, se resuelve y se integra. Con la creación artística se amplían las experiencias humanas, que, al ser convertidas en un objeto, son más fáciles de racionalizar posteriormente. En la actualidad, la metáfora es uno de los recursos más importantes del pensamiento creativo, y se encuentra siempre presente en el acto creativo en arteterapia.

Catalina Rigo (2004) señala que la exploración del entorno conduce a intentos de resolver problemas. De este modo, el arteterapia presenta una valiosa aportación, ya que la creación artística supone una herramienta para practicar la toma de decisiones y la resolución de problemas. Por su parte, Gata (2005) establece que la actividad artística, entendida como un medio para facilitar la expresión de contenidos emocionales, puede ayudar a las personas a abordar con mayor claridad sus problemas. Como un ejemplo de aplicación, Wiselogle (2007) describe el taller de arteterapia «Drawing out conflict» diseñado para mediar en el conflicto, donde quienes participan aprenden a elaborar sus conflictos a través del proceso creativo.

\section{Ayuda en la superación de experiencias dolorosas}

Kaplan (2007), siguiendo a Wiesel y Slater (2007), subraya el papel de los medios visuales para traer a la conciencia y exteriorizar las experiencias dolorosas. Ante situaciones traumáticas, el proceso artístico es importante para observar e identi- 
ficar las emociones difíciles así como los mecanismos de supervivencia que son saludables. Kramer (1992) sostenía que, desde el carácter simbólico de la expresión plástica se hace frente a las situaciones traumáticas, con la reelaboración de las experiencias desde el arteterapia se disminuye la ansiedad, se fortalece el yo y promueve la maduración.

\section{Disminuye las conductas conflictivas}

En un proyecto de integración dirigido a menores de un barrio marginal, Dolores Callejón (2005) constata, entre otras evidencias, que por medio del arteterapia se produce la disminución de las conductas agresivas y conflictivas entre quienes participan. Además aumenta la facilidad de conversación y emitir una opinión, y en general, de una mejor capacidad de cooperación.

Beatriz López (2004) afirma que las actividades creativas juegan un papel esencial para favorecer la expresión espontánea, la comunicación, la percepción, la organización espacio-tiempo, etc. De esta forma, el arteterapia puede canalizar la agresividad hasta que se revele su origen y pueda ser tratado el problema, proporcionando «oportunidades para poder utilizar las acciones destructivas en una forma creativa» (López, 2004)

\section{La creatividad como capacidad}

El arte como empowerment postula que la creación artística puede ser una vía para explorar las capacidades internas y potenciar los recursos propios que permitan gestionar y dirigir la vida propia. Serrano-Martínez (2016) nos muestra como el arte puede ser aplicado en un contexto social urbano como instrumento de empoderamiento y visibilización poniendo como ejemplo el Festival de asalto en Zaragoza. En su trabajo concluye que sería positivo poner en valor el arte como una herramienta aplicada al cambio social y como instrumento para el Trabajo Social. Desde una perspectiva de género, la práctica del arteterapia con mujeres ha revelado su potencial como un medio para empoderar su subjetividad. La expresión creativa permite accede al mundo interior, conocerse, identificarse, entender su singularidad, generando la identificación de sus deseos y necesidades, y la comprensión a si misma (Guiote, 2013). El arteterapia potencia el empoderamiento personal, base para acceder a los niveles de poder interpersonal y comunitario.

\section{b) Área de desarrollo social}

\section{Desarrolla las habilidades sociales y el interés social}

Edith Kramer destacó la importancia de la atención a las interacciones surgidas en el proceso de intervención terapéutica en las sesiones grupales. Este tipo de sesiones favorecen las relaciones interpersonales, y esto ayuda a mejorar sus habilidades sociales. Cuando el arteterapia se dirige a familias, el proceso alienta a las personas a tomar conciencia de sí mismas y promueve un sentimiento de pertenencia que proporciona la confianza necesaria para el desarrollo del interés social (Sutherland, 2011). El trabajo dialógico a través del arte favorece la socia- 
lización, hecho relacionado con los procesos de integración consigo mismo/a y con el medio. (Callejón, 2005).

\section{Mejora la expresión y la comunicación}

El hecho de recurrir a formas de comunicación alternativas a la palabra, como lo es la expresión artística, ayuda a compartir las vivencias más personales. Según M.D. López (2011), estimular la expresión y la comunicación mediante las actividades artísticas puede proporcionar habilidades enriquecedoras a cualquier persona, pero resulta especialmente interesante cuando el idioma, la edad, la cultura o el estado psicofísico resulten un obstáculo.

El arteterapia trata de comprender y responder adecuadamente a los mensajes no verbales y facilitar el aprendizaje de nuevas formas de comunicación más elaboradas (Torrado, 2005). Y está especialmente indicado para niños y niñas, ya que utiliza una forma de comunicación que les resulta muy próxima y asequible según sostiene B. López (2004). En el caso de la discapacidad, es frecuente que sufran trastornos del lenguaje o de movimiento, pudiéndoles causar aislamiento e inadaptación social. El arte les proporciona una alternativa al lenguaje hablado para comunicarse, y por tanto para relacionarse.

\section{Aportaciones del arteterapia al desarrollo la intervención social}

\section{Facilita del cambio}

Cuando una persona encuentra un medio para expresar algo que no puede verbalizar, este hecho en sí mismo es una toma de conciencia que facilita el cambio personal. Cuando la persona arteterapeuta acompaña, y es testigo de esta expresión, agrega significado y favorece el proceso. El arte se concibe por tanto como un vehículo de cambio de actitud (Torrado, 2005). González y García (2005) constatan, desde su experiencia con menores en un centro de protección, que el arte enfocado como terapia facilita un cambio de comportamiento de quienes presentan conductas desadaptativas.

Noemí Martínez y Marián López Fdez. Cao (2004), en su introducción al libro Arteterapia y educación, exponen que el arteterapia desmonta las defensas que dificultan tanto el proceso de subjetivación como el vínculo de la persona con sus grupos y su pertenencia a la sociedad. Por su parte, Sutherland (2011) afirma que una de las ventajas del arteterapia con familia es que, frente a la fortaleza de las defensas del grupo, aporta una forma de intervenir poco amenazante que facilita exteriorizar los conflictos familiares. El entorno seguro y de confianza que se crea en la sesión de arteterapia favorece el cambio de las personas clientes.

\section{Es una experiencia gratificante}

Cuando la experiencia creativa resulta gratificante por sí misma, lleva a la persona cliente a motivarse con el proceso terapéutico. La satisfacción personal actúa como refuerzo interno y mejora el compromiso con la actividad y por tanto la continuidad de la intervención. Como señalan Rico e Izquierdo (2011), la experiencia demuestra 
que las técnicas relacionadas con el proceso artístico resultan atractivas, especialmente entre los niños y las niñas.

\section{Facilita la proyección a futuro}

Besa y Ponce (2014) exponen que a partir de la creación artística la persona puede ubicarse a si misma de forma distinta. Tomando como referencia la potencialidad, y no el déficit, la persona acentúa sus habilidades dejando a un lado el diagnóstico, en muchos casos estigmatizador. Desecha la etiqueta que le condena, lo que en la práctica enriquece el autoconcepto y la imagen de sí. De este modo, el arteterapia da la posibilidad a las personas, con enfermedad mental o discapacidad por ejemplo, de proyectarse a futuro obviando el estigma social asociado a su situación, y desarrollando su propia subjetividad desde sus habilidades y potencialidades. El arte, entre otras cosas, ofrece a las personas otros modos y posibilidades de ser, de sentir y de expresarse (Carnacea, 2014). Les permite imaginarse en otra situación, y proyectar un camino hacia un futuro de bienestar.

\section{Espacio de detección de problemáticas y necesidades}

Dado que por medio del lenguaje plástico las personas pueden comenzar a expresar esas preocupaciones que han estado ocultas (Martínez y López, 2004), el espacio de arteterapia se convierte en un espacio donde poder detectar riesgos sociales y situaciones de vulnerabilidad enmascaradas a veces por el miedo o por las urgencias vitales.

El arteterapia se ofrece un espacio para exteriorizar pensamientos, emociones, sentimientos que quedaban bajo la autoridad de las palabras, y que ahora son compartidas a través de la «proyección». Esta posibilidad de proyectar, de reflejar en la producción plástica lo que resulta complicado compartir con el lenguaje verbal, es un recurso clave en arteterapia que aporta una información ocultada, consciente o inconscientemente pero importante (López, 2011; Duncan, 2007). Desde un lenguaje simbólico, la actividad artística permite expresar, problemas o conflictos personales sin el control consciente de la comunicación verbal, y es esta posibilidad exteriorizarlos permite detectarlos. El arteterapia proporciona de este modo una información valiosa para actuar de forma preventiva e iniciar los procesos de intervención más ajustados a las necesidades de cada persona. Como apunta B. López (2004), a través del lenguaje plástico se pueden detectar y prevenir situaciones tan importantes y urgentes como el maltrato en la infancia.

\section{Conclusiones}

Más allá de las necesidades fisiológicas y de seguridad a las que Maslow hace referencia en su pirámide, encontramos la necesidad de esa fuente de apoyo emocional que es la red social, y que el trabajo en lo social ha de tomar en consideración en beneficio de una intervención social integral y normalizadora. La persona no puede y no debe encontrarse atendida únicamente en sus necesidades materiales, la salud emocional contribuye positivamente a mejorar otras áreas de desarrollo como es el área personal y social, objetivos del trabajo social. Como recurso en la intervención social, la actividad arteterapéutica facilita el ajuste de la realidad interna con 
la realidad externa para alcanzar una respuesta más saludable y adaptativa ante los elementos desafiantes de la vida.

La exclusión social es un proceso que se inicia con la desigualdad social, una condición que hace a las personas vulnerables. La sucesiva desvinculación del entorno social que lleva a los márgenes del sistema es un continuo de vivencias traumáticas que las personas en situación de vulnerabilidad deben manejar. La gestión de estas experiencias requiere la implicación de las personas afectadas, han de ser protagonistas de un proceso de resiliencia que funcione como factor de protección. En este camino, la red social y familiar tiene la función primordial de ayudar las personas que sufren la desigualdad a superar los sucesos negativos, las frustraciones y la fractura social y personal, siendo el tejido social un soporte que contribuye a afrontar la condición de riesgo. La ausencia de esta red, especialmente la que proporciona el entorno familiar, como contexto básico de socialización, correlaciona con el incremento del riesgo de exclusión. En un contexto social donde las relaciones familiares se fragmentan y lo comunitario ha dejado paso a la individualidad, la red de apoyo social se reduce.

La contribución del arteterapia al desarrollo personal y social recogida, entronca con los objetivos que los servicios sociales estipulan en sus actuaciones. La intervención social busca valorar las necesidades de las personas en las diferentes áreas (personal, las relaciones afectivas familiares, social y formativa-laboral) e incrementar el apoyo y protección social de las personas fortaleciendo la red de apoyo social y contribuyendo a la coordinación de recursos comunitarios. Además de acompañar y apoyar en su itinerario de desarrollo personal, la intervención se orienta a proporcionar recursos que permitan avanzar en él, y experiencias de referencia que apoyen tanto el proceso de construcción de un proyecto personal como el proceso se socialización. El arteterapia proporciona un contexto seguro para esa construcción.

La vulnerabilidad se asocia a la exposición de una persona o un grupo a sufrir un daño, pero también a la capacidad de evitar o minimizar los efectos traumáticos de esas circunstancias vitales. Capacitar para la adecuada gestión los riesgos es una estrategia de inclusión necesaria para sobrellevar la desventaja social. Desarrollar recursos para afrontar las adaptaciones requeridas, en un contexto cambiante, permite limitar las rupturas sucesivas que preceden a la exclusión social, y se convierte en un factor de protección fundamental. El arteterapia permite abordar en la intervención estas dimensiones de la vulnerabilidad restaurando las capacidades de afrontamiento. La persona se dota de estrategias para sobrevivir a los impactos y participar activamente en el proceso de reconstrucción de los vínculos sociales perdidos.

Ante la falta del apoyo emocional que proporcionan los vínculos sociales y familiares adecuados, y que atenúan la dureza de la desigualdad social, el arteterapia se convierte en un recurso para acompañar a las personas en situación de vulnerabilidad desde el enfoque integral que demanda la atención social. Facilita la gestión de las amenazas tratando las vivencias que impactan de forma profunda en las personas. También favorece las capacidades adaptativas, fundamentales para afrontar la indefensión y la incertidumbre al que se enfrentan las personas vulnerables en un entorno socioeconómico cambiante. El carácter simbólico de la producción artística les permite expresar y enfrentarse a los shocks antes de poder hablar de ellos. Al elaborar el conflicto y enfrentarse a sus temores desde el terreno de lo simbólico se fortalece el yo y disminuye la ansiedad que subyace a las dificultades sociales. Y con la sanación emocional y la superación de las experiencias disruptivas, se puede fundamentar una 
intervención que proporcione aprendizajes duraderos que resulten protectores frente a los riesgos de la exclusión social.

El arteterapia constituye un recurso válido de prevención e intervención en el ámbito de los servicios sociales. Como se ha señalado anteriormente, el arteterapia proporciona elementos de interés y logros que confluyen con los objetivos de la intervención social, facilitando el cambio personal y social que mejore la vida de las personas y les mecanismos protectores frente a los riesgos sociales. El arteterapia contribuye al desarrollo personal al mejorar la gestión de las emociones, un control y conocimiento sobre sí que disminuye las conductas conflictivas. El arteterapia ayuda a la superación de vivencias angustiosas. La expresión y la elaboración emocional de las dificultades y problemáticas a las que se exponen, permite a las personas afrontarlas con mayor fortaleza y aprender a gestionar recursos que les son propios. La capacidad de afrontamiento y gestión emocional que se adquieren funcionan como recursos que facilitan la resolución de problemas.

La vía de dialogo que se abre gracias al lenguaje artístico en la sesión de arteterapia mejora la expresión y la comunicación, estableciendo un vinculo interpersonal en las sesiones que favorece las relaciones sociales. Esta práctica relacional positiva motiva el interés social y entrena y mejora las habilidades sociales. Sobre la base de un desarrollo personal enriquecedor, a través del conocimiento de sí y sus fortalezas se fundamenta un desarrollo social positivo que contribuye a la inclusión social. Es la base para construir la intervención preventiva y protectora frente a la agresividad de los riesgos sociales.

Si los servicios sociales se orientan a un modelo de intervención que proponga cambios duraderos basados en el potencial y la capacidad de las personas para participar y dirigir su propia trayectoria vital, el arteterapia es un recurso que apoya ese enfoque de trabajo y una metodología facilitadora de la intervención social interdisciplinar.

Indudablemente las personas que acuden a los servicios de atención social pasan por situaciones personales y familiares de dificultad. Es un compromiso básico del Trabajo Social mejorar la vida de estas personas proporcionándoles aquellos recursos materiales, sociales y personales necesarios para retomar su proyecto vital. La nuevas tendencias en trabajo social entienden que, más allá del trabajo administrativo y los servicios urgentes y puntuales, las personas usuarias de los servicios sociales deben reparar el daño que ha producido la vivencia. El arteterapia puede funcionar en este contexto como un recurso preventivo que permita fortalecer a las personas, a las familias, para hacer frente a los cambios continuos y retos que caracterizan la sociedad actual. Quizá no se pueda evitar muchas de las dificultades que las personas que acuden a servicios sociales puedan encontrar, pero se pueden proporcionar estrategias útiles y transferibles a situaciones futuras, movilizando recursos propios a través del arteterapia, aprendizajes que proporcionen un apoyo, que funcionen como un factor de protección. En definitiva, que responda a los propósitos de la intervención social de prevención y rehabilitación buscando el bienestar de las personas y su participación en la comunidad.

Al atender las necesidades emocionales, el arteterapia ayuda a las personas en situación de vulnerabilidad a afrontar los procesos traumáticos, las inseguridades y las rupturas sociales, a situarse frente a la dificultad de otra manera, fortalecida y en cierto modo con poder y control sobre sus propios asuntos. Conecta a la persona consigo misma para poder conectarla con el entorno social. La vulnerabilidad, como 
«antesala de la exclusión», provoca en muchos casos un recorrido vital errático, no lineal, que necesita una intervención basada en la prevención, al tiempo que responda a las prácticas sociales basadas en las capacidades. Frente a la incapacidad para acceder a los mecanismos de desarrollo personal y de inserción sociocomunitaria que mencionaba Subirats (2004), en referencia a la exclusión social, la terapia a través del arte es habilitante y ayuda a resolver los conflictos profundos. De este modo, el arteterapia se puede asociar de forma válida al trabajo social enriqueciéndose mutuamente en una intervención integral e integradora.

\section{Referencias bibliográficas}

Besa, D. y Ponce, V. (2014). Arte terapia grupal infanto-juvenil en centro comunitario de salud mental familiar. PRAXIS. Revista de Psicología 25, 7-35

Camarena, J. (2015). Arteterapia como profesión. Integración en el NHS. VII Jornade DÁrteterapie en salut, educació et comunitat. 8 al 9 de mayo. Barcelona.

Carnacea, M. A. (2014). Arte, Intervención y Acción Social. Hacia la Construcción de Comunidades Inclusivas. VI Congreso Iberoamericano de Cultura. 11 al 13 de abril. San José.

Castel, R. (2002). La metamorfosis de la cuestión social. Barcelona: Paidos Iberica.

Castel, R. (2014). Los riesgos de exclusión social en un contexto de incertidumbre. Revista Internacional de Sociología, 72 (1), 15-24

Castillo, R, Sostegno, R y López-Arostegi, R. (2012). Arte para la inclusión y la transformación social. Bilbao: Observatorio del Tercer Sector de Bizkaia.

Del Pino, E y Rubio, MJ. Ed.(2013). Los estados de bienestar en la encrucijada. Políticas sociales en perspectiva comparada. Madrid: Tecnos.

Duncan, N. (2007). Trabajar con las Emociones en Arteterapia. Arteterapia - Papeles de arteterapia y educación artística para la inclusión social, 2, 39-49. Recuperado de https:// revistas.ucm.es/index.php/ARTE/article/view/ARTE0707110039A

Fernández, B. (2010). Menores no acompañados: de la vulnerabilidad social a la invisibilidad institucional. Zerbitzuan 48,145-153

Flores, F. (2014). Vulnerabilidad y representación social de género en mujeres de una comunidad migrante. Península, 9(2), 41-57. doi:10.1016/S1870-5766(14)71799-4

Guiote, A. (2013). Arteterapia como acompañamiento para la construcción y empoderamiento de la subjetividad femenina. Investigaciones Feministas, 4,171-199

Hernández-Echegaray, A. (2016). Tendencias del Trabajo Social en el ámbito de los Servicios Sociales desde la perspectiva profesional. Estudio Delphi. Comunitania. 12 doi: 0.5944/ comunitania.12.3

Kaplan, F. (2007). Art Therapy and Social Action: Treating the World's Wounds. Londres: Jessica Kingsley Publishers.

López B. (2004). Arteterapia. Otra forma de curar. Educación y futuro: revista de investigación aplicada y experiencias educativa, 10,101-110. Recuperado de https:/dialnet.unirioja.es/ servlet/articulo?codigo $=2044648$

López M.D.( 2011). Arteterapia y Museos. Puntos de encuentro. Revista Arte y políticas de identidad , 4, 127-136. Recuperado de http://revistas.um.es/api/article/ view/146041/130451

López, M. Coord. (2006). Creación y posibilidad. Aplicaciones del arte en la integración social. Madrid: Editorial Fundamentos. 
Malgesini, G. (2011). Informe anual sobre vulnerabilidad social 2010. Madrid: Cruz Roja Española.

Malgesini, G. (2011). Informe anual sobre vulnerabilidad social. Madrid:Cruz Roja

Martínez, N. y López, M. Coord. (2004). Arteterapia y educación. Madrid: Comunidad de Madrid.

Moreno, A. (2006). El arte: punto de encuentro. Arteterapia. Encuentros con la expresión 1, 57-60.

Mundet A., Beltrán A.M. Y Moreno A. (2015). Arte como herramienta social y educativa. Revista Complutense de Educación, 26 (2), 315-329. doi: 10.5209/rev_RCED.2015.v26. n2.43060

Muñoz, M. (2002). La educación a través del arte en la educación social.

Pedagogia social: revista interuniversitaria, 9, 287-298.

Pizarro, R. (2001). La vulnerabilidad social y sus desafíos: una mirada desde América Latina. Santiago de Chile: CEPAL. Recuperado de https://repositorio.cepal.org/bitstream/ handle/11362/4762/S0102116_es.pdf

Ramirez, U. Gomez, S., Rodriguez, R. (2017) Tendencias contemporáneas en relación con la investigación y la intervención en trabajo social. Tabula Rasa, 27, 45-68. doi: $10.25058 / 20112742.456$

Rico, L. e Izquierdo, G. (2010). Arte en Contextos Especiales. Inclusión Social y Terapia a través del Arte. Trabajando con Niños y Jóvenes Inmigrantes. Arteterapia: Papeles de arteterapia y educación artística para la inclusión social , 5,153-167. Recuperado de https://revistas.ucm.es/index.php/ARTE/article/view/ARTE1010110153A/8735

Ruiz Rivera, N. (2012). La definición y medición de la vulnerabilidad social. Un enfoque normativo. Investigaciones Geográficas (Mx), 77, 63-74. Recuperado de http://www. redalyc.org/articulo.oa?id $=56923353006$

Sanchez, A. Jiménez, M. (2013) . Trabajo Social Global. Revista de Investigaciones en Intervención Social, 3(4)133-15.

Serrano-Martínez, C. (2016). El arte urbano como instrumento de empoderamiento y visibilización. El Festival de Asalto. Comunitania: Revista Internacional de Trabajo Social y Ciencias Sociales, 11, 9-26. Recuperado de http:/www.comunitania.com/ cecilia-serrano-Martínez-el- arte-urbano-como-instrumento-de-empoderamiento-yvisibilizacion-el-festival-de-asalto

Serrano, A. y Carmona E. (2014). Zoom al interior: la exploración de la mirada a través de la imagen y el lenguaje audiovisual y sus posibilidades para la intervención con niños y adolescentes desde el arteterapia y el arte. Arteterapia: Papeles de arteterapia y educación artística para la inclusión social, 9, 327-347. doi:10.5209/rev_ARTE.2014.v9.47499

Subirats, J et al. (2005). Análisis de los factores de exclusión social. Madrid: Ministerio de Trabajo y Asuntos Sociales.

Sutherland, J. (2011). Art therapy with families. The Journal of Individual Psychology, 67 (3), 292. Recuperado de http://connection.ebscohost.com/c/articles/74566908/arttherapy-families. 